\title{
Improving the imaging ability of ultrasound-modulated optical tomography with spectral-hole burning
}

Xiao Xu, Honglin Liu, Lihong V. Wang

Xiao Xu, Honglin Liu, Lihong V. Wang, "Improving the imaging ability of ultrasound-modulated optical tomography with spectral-hole burning," Proc. SPIE 7948, Advances in Photonics of Quantum Computing, Memory, and Communication IV, 794803 (10 February 2011); doi: 10.1117/12.873999 


\title{
Improving the imaging ability of ultrasound-modulated optical tomography with spectral-hole burning
}

\author{
Xiao Xu*, Honglin Liu, Lihong V. Wang \\ Optical Imaging Laboratory, Dept of Biomedical Engineering, Washington Univ. in St. Louis, \\ 1 Brookings Dr., St. Louis, MO USA 63130-1097
}

\begin{abstract}
Ultrasound-modulated optical tomography is a hybrid imaging technique based on detection of the diffused light modulated by a focused ultrasonic wave inside a scattering medium. With the combined advantages of ultrasonic resolution and optical contrast, UOT is ideal for deep tissue imaging. Its growth in popularity and application, however, is hindered by the low efficiency in detecting the modulated diffused photons. Research activities on UOT have therefore been centered on improving its signal detection efficiency by exploring various technical solutions. A prime example is the use of spectral-hole burning (SHB) in UOT. By applying SHB crystal as a spectral filter, one modulation sideband of the diffused light can be efficiently selected while all the other frequency components are strongly suppressed. Immune to both the spatial and temporal incoherence of the signal with a high enough on/off ratio, SHB can boost the UOT imaging ability dramatically and push it towards practical applications. We compare SHB with the other technologies that have been applied to UOT, and identify the unique features that make SHB a preferable tool for UOT. We also discuss the desired improvements from the SHB side, which will help UOT pave the way from research to everyday life.
\end{abstract}

Keywords: Optical Imaging, Optical Tomography, Tissue Optics, Spectral-Hole Burning, Ultrasound-modulated Optical Tomography, UOT, Optical Memory

\section{INTRODUCTION}

\subsection{Ultrasound-modulated optical tomography}

Optical imaging of soft biological tissue is a non-invasive, nonionizing, and functional imaging modality, which makes it highly desirable in the biomedical field. ${ }^{1}$ The photon energy used in optical imaging is typically $\sim 2 \mathrm{eV}$ for $\lambda=500 \mathrm{~nm}$, which is safe for biological molecules as compared with the photons used in X-ray imaging which is $\sim 50 \mathrm{KeV}$. Because its contrast is based on optical properties (i.e., absorption and scattering) of the tissue, optical imaging can provide better soft-tissue contrast than the ultrasound imaging which is based on mechanical contrast. The optical absorption and scattering properties in the visible and near infrared wavelengths are intrinsic indicators of tissue abnormalities and functions since they are determined by the molecular constituents of tissues and the electronic and/or vibrational structures at the molecular level. ${ }^{2-4}$ For example, cancerous tissues manifest significant architectural changes at the cellular and sub-cellular levels, which result in changes to the optical scattering properties. ${ }^{5}$ Angiogenesis and hypermetabolism, which are characteristic of cancers, result in changes to the optical absorption properties. ${ }^{6}$ Therefore, optical imaging holds great promise for early cancer detection. Other important physiological parameters can also be measured by optical imaging such as the oxygen saturation of hemoglobin, which makes optical functional imaging possible. ${ }^{7}$

However, pure optical imaging lacks good spatial resolution in deep tissue because of strong optical scattering in the visible and NIR wavelengths. For imaging modalities that detect ballistic or quasi-ballistic photons as imaging signal, such as time-gated imaging ${ }^{8-10}$ and optical coherence tomography ${ }^{11,12}$, the detected photons experience minimal scattering inside tissues and carry spatial information, but the signal strength decays exponentially with increasing tissue thickness. These imaging modalities are therefore not suitable for deep tissue imaging. For diffuse optical imaging modalities that collect diffused photons transmitted through thick tissues as imaging signal, the diffused light decays more slowly with thick tissues, but the spatial information is lost in diffuse photons because multiple scattering randomize light propagation after several scattering mean free paths. At $\lambda=500 \mathrm{~nm}$ the mean scattering free path $l_{S}=0.1$

*xxu@biomed.wustl.edu; phone 1314 935-9587

Advances in Photonics of Quantum Computing, Memory, and Communication IV, edited by Zameer UI Hasan, Philip R. Hemmer, Hwang Lee, Charles M. Santori, Proc. of SPIE Vol. 7948, 794803 - (C) 2011 SPIE · CCC code: 0277-786X/11/\$18 - doi: 10.1117/12.873999 
$\mathrm{mm}$ in human breast, while $l_{S}=50 \mathrm{~mm}$ for photons at X-ray wavelength used in medical diagnosis. In diffuse optical imaging, convoluted image reconstruction is needed to recover the spatial distribution of the relevant optical properties. Since this is usually an ill-posed inverse problem, the image resolution and the image quality is not good enough.

To take advantage of the high optical contrast and overcome the poor spatial resolution for thick tissues in optical imaging, the use of ultrasound has been explored, which resulted in several hybrid imaging modalities such as photoacoustic imaging ${ }^{13-15}$ and ultrasound-modulated optical tomography (UOT) ${ }^{16}$. Because acoustic scattering is 1000 times less than optical scattering in soft biological tissues, ultrasonic waves provide spatial resolution in these hybrid imaging modalities. In UOT, a focused ultrasonic wave is used to modulate the diffused light inside biological tissues. The ultrasonically modulated (or encoded) photons are frequency shifted by the ultrasonic frequency and can be discriminated from the un-modulated background light. The ultrasonic focus determines the spatial resolution for the ultrasonically modulated photons. Thus UOT has the combined advantages of optical contrast and ultrasonic resolution.

\subsection{Signal detection in UOT}

In UOT, a focused ultrasound beam interacts with diffused light by means of phase modulation inside a biological sample. The intensity of the ultrasonically modulated (or tagged) light at the detector is proportional to the fluence of the diffused light probed by the ultrasonic wave. By scanning the ultrasound beam through the sample, a tomographic image of the light fluence can be constructed, which can be used to recover the spatial distribution of optical properties which are of biomedical interests.

The development of UOT has been chronicled by the development of its signal detection methods (Table 1). When ultrasound modulation of diffused light was first experimentally demonstrated by Marks et al. ${ }^{17}$ single element detector (photomultiplier) and Fabry-Perot interferometer were used for signal detection in the time and spectral domain. Leveque et al. ${ }^{18}$ implemented parallel speckle detection method to improved the signal to noise ratio (SNR), where a CCD camera was used as a detector array with a source-synchronized lock-in technique. Sakadzic and Wang ${ }^{19}$ used single ultrasound pulses and a confocal Fabry-Perot interferometer to obtain microscopic UOT images in biological tissues with resolution scalable to ultrasound wavelength $(<100 \mathrm{um})$ at imaging depths $>3 \mathrm{~mm}$. Murray et al. ${ }^{20}$ used photorefractive crystal (PRC) based detection system to enhance the detection efficiency.

Table 1. Different signal detection methods in UOT.

\begin{tabular}{|l|l|l|}
\hline \multicolumn{1}{|c|}{ Detection methods } & \multicolumn{1}{|c|}{ Main features } & \multicolumn{1}{c|}{ First reported by } \\
\hline $\begin{array}{l}\text { Single element detector } \\
\text { (photo diode, PMT, etc.) }\end{array}$ & $\begin{array}{l}\text { Single speckle grain; real time response; } \\
\text { photon starving }\end{array}$ & $\begin{array}{l}\text { W. Leutz } \text { et al. }{ }^{21} \text {, L. Wang } \text { et al. } \\
\text { M. Kempe } \text { et al. }{ }^{22}\end{array}$ \\
\hline $\begin{array}{l}\text { Parallel detection } \\
\text { (CCD) }\end{array}$ & $\begin{array}{l}\text { Parallel detection of multiple speckle grains; } \\
\text { improved etendue; slow CCD exposure speed; } \\
\text { photon starving }\end{array}$ & S. Leveque et al. \\
\hline Fabry-Perot interferometer & $\begin{array}{l}\text { Spectral filtering; immune to speckle; small } \\
\text { etendue; slow speed }\end{array}$ & W. Leutz et al. S Sakadzic et al. \\
\hline $\begin{array}{l}\text { Photorefractive detection } \\
\text { (photorefractive material) }\end{array}$ & $\begin{array}{l}\text { Adaptive, dynamic beam splitter; increased } \\
\text { etendue; spectral filtering; }\end{array}$ & TW Murray et al. \\
\hline $\begin{array}{l}\text { Spectral-hole burning } \\
\text { (SHB crystal) }\end{array}$ & Spectral filtering; largest etendue; & Y. Li et al. ${ }^{23}$ \\
\hline
\end{tabular}

The central theme in the development of the UOT signal detection methods is to improve the SNR and the system etendue, which are the main difficulties in UOT and are inherently related to the mechanism of ultrasonic modulation of coherent laser light in a scattering medium. ${ }^{24,25}$ First, because the ultrasonic focal volume is small compared to the entire light diffusion volume, the modulation depth $m$-defined as the ratio of the ultrasound-modulated light intensity to the un-modulated light intensity - is low. Secondly, multiple scattering results in a speckled wavefront of the diffused light. The average grain size of the speckle sets the upper limit of the spatial coherence area when both of the ultrasoundmodulated and un-modulated components fall on the photo detector surface. For a single speckle grain, the heterodyne mixing of the two components has an etendue $<\lambda^{2}$ (where $\lambda$ is the light wavelength) because the two wavefronts are not perfectly matched since the two components follow independent statistics in diffusion. When the detector surface is large 
compared to the average speckle grain size $(\sim \lambda \mathrm{D} / \mathrm{L}$, where $\mathrm{D}$ is the dimension of light emanating surface, and $\mathrm{L}$ is the distance between the source and the detector), multiple speckle grains will be integrated which results in a reduced effective modulation depth $m_{e f f}=\langle m\rangle / \sqrt{N},{ }^{22}$ where $\mathrm{N}$ is the number of grains. This leads to a trade-off between the measured $m_{\text {eff }}$ and the system etendue which can be defined as $G=\iint_{\Omega, S} d \Omega_{S} d S$, where $d \Omega_{S}$ is the solid angle subtended by the light emanating surface when viewed from the surface element $d S$ of the detector. A system with an improved etendue will collect more signal light, but will result in a decreased $m_{\text {eff }}$ because of an increased number of the speckle grains $N$; and vice versa. An additional complication in the en vivo imaging of biological tissue is the speckle decorrelation caused by the internal movement of the scattering medium such as Brownian motion. This limits the coherence detection time to within the speckle decorrelation time of the tissue, usually on the order of $1-100$ milliseconds.

\subsection{Spectral-hole burning}

The various methods developed for UOT signal detection address the difficulty using different approaches. Each method has its unique advantages, but has inevitably some drawbacks. Spectral-hole burning (SHB), as one of the latest developments, offers great promises to improve the signal detection for UOT with little sacrifice, thereby push this imaging modality to practical application.

The use of SHB as a narrow-band pass absorptive filter has been reported in other fields ${ }^{26}$. In UOT, with SHB assisted detection, a spectral hole is first burned in the SHB crystal at the frequency of the ultrasound-modulated light. When the diffused light from the UOT study sample is transmitted through the SHB crystal, the ultrasound-modulated component will be transmitted with little absorption while the un-modulated component will be strongly absorbed. In the ideal case, passing through the SHB crystal will filter out almost all of the un-modulated light. Only the modulated light will reach the photo detector, thereby removing the spatial and temporal coherence constraints imposed by heterodyne detection. Furthermore, its ability to parallel process numerous speckles grains makes it possible to increase the system's etendue.

\section{EXPERIMENTAL}

\section{1 $\mathrm{Tm}^{3+}$ : YAG crystal used in SHB}

The SHB crystal used in our experiment is a 2.0 -atomic\%-doped $\mathrm{Tm}^{3+}: \mathrm{YAG}$ crystal, with dimensions of $10 \times 9 \times 2.5 \mathrm{~mm}^{3}$. It has an absorption peak at $\lambda=793.38 \mathrm{~nm}$, corresponding to the ${ }^{3} \mathrm{H}_{6} \rightarrow{ }^{3} \mathrm{H}_{4}$ transition of the Tm ${ }^{3+}$ ions (Figure 1) at temperatures below $4.7 \mathrm{~K}$. This wavelength is within the preferred NIR biological imaging window. The homogeneous linewidth of the crystal is $\sim 150 \mathrm{kHz}$ and the inhomogeneous absorption linewidth is $\sim 25 \mathrm{GHz}$. The spectral hole burned by the pump light from a Ti-Sapphire laser in the experiment has a FWHM around $710 \mathrm{kHz}$, narrow enough to remove the un-modulated light which is shifted by $5 \mathrm{MHz}$ (the ultrasonic frequency) from the ultrasoundmodulated light. The spectral-hole's life time is $\sim 10 \mathrm{~ms}^{15}$ due to the existence of a shelving middle state ${ }^{3} \mathrm{~F}_{4}$, which make it possible in UOT experiments to switch between the burning the spectral-hole and detecting UOT signal.

\subsection{Experimental setup for SHB-UOT}

Figure 2 schematically shows our experimental setup for UOT with SHB. The experiment is carried out in two steps. First a spectral-hole is burned in the $\mathrm{Tm}^{3+}$ :YAG crystal by a pump beam, then the signal light from a study sample is transmitted through the SHB crystal and detected by a photodetector. The light source is a CW Ti:Sapphire laser (Coherent MBR110E) operating at $793.38 \mathrm{~nm}$ with a linewidth of $\sim 181 \mathrm{kHz}$ and output power of $2 \mathrm{~W}$. To burn the spectral-hole, an acousto-optic modulator (AOM, IntraAction AOM 802-A1), AOM1, working at $70 \mathrm{MHz}$, is turned on for $3.3 \mathrm{~ms}$ to steer light from the laser into its first diffraction order as the pump beam. After collimation, the pump beam has a diameter of $9 \mathrm{~mm}$ diameter and optical power of $900 \mathrm{~mW}$ to illuminate the crystal on its $10 \times 9 \mathrm{~mm}^{2}$ side. After the 3.3 ms pump period, AOM1 is turned off. Then the laser beam is sent through the first diffraction order of AOM2 which works at $75 \mathrm{MHz}$, and illuminates the scattering sample. The diffused light is modulated by a traveling ultrasound burst of 5-cycles sinusoidal wave, emitted by a focused transducer (Panametrics-NDT A326S; central frequency $=5$ $\mathrm{MHz}$; focal length $=16.2 \mathrm{~mm}$; focal width $=0.5 \mathrm{~mm}$ ). The diffused light out of the sample is transmitted through the SHB crystal which is placed inside the cryostat chamber. The modulated component of the signal light was spectrally filtered by the SHB crystal before it is detected by a photomultiplier tube (PMT, Hamamatsu R928). The output from the 
PMT is fed through a preamplifier (Stanford Research SR560) and coupled into a digitizer (Gage CompuScope 14200) for imaging analysis.

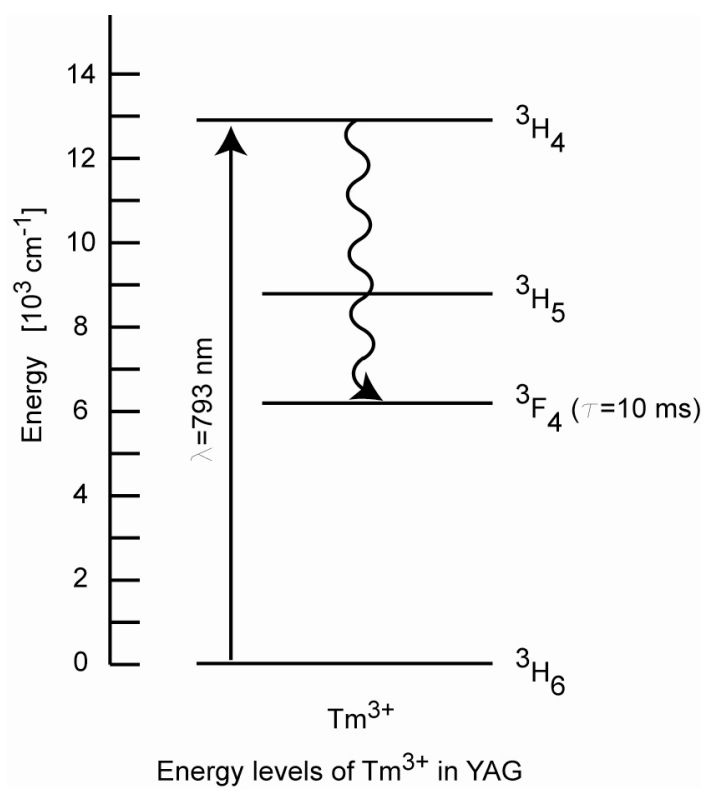

Figure 1. Energy transition in $\mathrm{Tm}^{3+}$ :YAG corresponding to $\lambda=793.38 \mathrm{~nm}$ absorption.

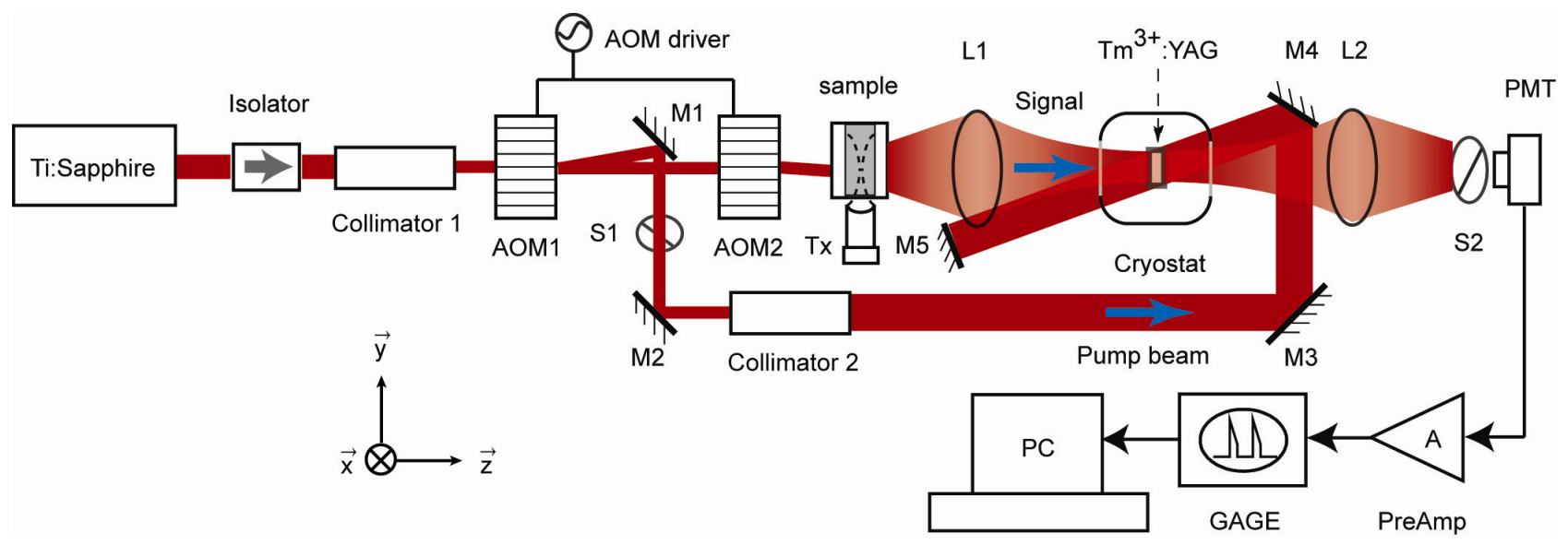

Figure 2. schematic of SHB-UOT experimental setup: Ti-Sapphire laser, Coherent MBR110; L1 - L6, lens; M1 M5, mirrors; AOM1, 2, acousto-optic modulators; S1, S2, mechanical shutters; Tx, ultrasound transducer; PMT, photomultiplier tube; PreAmp, preamplifier; GAGE, $200 \mathrm{MHz}$ data acquisition card; PC, personal computer.

\section{RESULTS}

\subsection{Imaging of thick tissue-mimicking sample}

Various biological samples have been imaged by the SHB-UOT system. ${ }^{10}$ Here we show the UOT image of a $40 \mathrm{~mm}$ thick tissue-mimicking sample. The sample was prepared by mixing 10-wt.\% porcine gelatin (Sigma G2500) and 1-wt.\% intralipid (Fresenius Kabi) in distilled water and molding the solution into a slab whose dimension was $100 \times 100 \times 40$ $\mathrm{mm}^{3}$ with the $40 \mathrm{~mm}$ thickness along the light propagation direction. The resulted sample had a reduced scattering coefficient of $\mu_{S}^{\prime}=0.7 \mathrm{~mm}^{-1}$ for $\lambda=793.38 \mathrm{~nm}$, comparable to that of human breast. Three absorbing cubes were embedded in the middle plane of the sample, with dimensions of $1 \times 1 \times 1 \mathrm{~mm}^{3}, 3 \times 3 \times 3 \mathrm{~mm}^{3}$, and $5 \times 5 \times 5 \mathrm{~mm}^{3}$, 
respectively. Their optical absorption coefficient is $\mu_{a}=10 \mathrm{~mm}^{-1}$ because of the addition of black India ink in these volumes.

Figure 3 shows a typical imaging result obtained from the SHB-UOT experiment, which was a reconstructed image of the three absorbing objects embedded in the sample after a raster scan (B-scan) of the sample along the $x$ axis, whereas at each $x$ position the temporal evolution of the UOT signal along the $y$ axis (the ultrasound propagation direction) constitutes the A-scan. The image shows very high contrast of the objects compared to the background matrix.
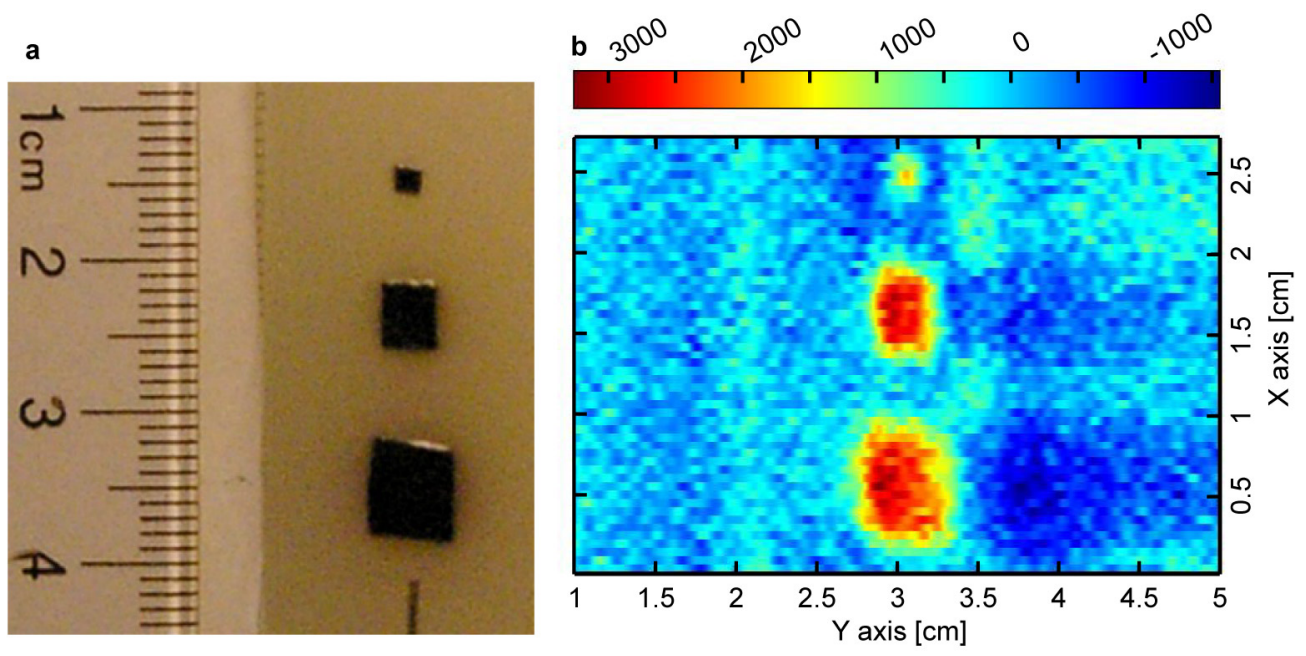

Figure 3. UOT image of the 3 absorbing objects embedded in the middle plane of a $40-\mathrm{mm}$ thick tissue-mimicking phantom. a. A photograph of the sample with the 3 embedded objects. b. A B-scan image of the 3 objects obtained from SHB-UOT.

\subsection{Harmonic imaging using SHB-UOT}

Another interesting feature of the SHB detection for UOT is that multiple spectral-holes can be burned and accessed that will transmit multiple sidebands of the ultrasound-modulated light. To demonstrate this, we investigated harmonic imaging using the SHB-UOT system.

The peak to peak pressure at the focus of the $5 \mathrm{MHz}$ ultrasound transducer in the experiment was measured to be $2 \mathrm{MPa}$. At this magnitude, the nonlinear effect in the ultrasonic waveform is already significant enough that the higher harmonics have come into play in the modulation of the diffused light inside the sample. Fourier analysis shows that the second harmonic frequency of a 5 -cycle $5 \mathrm{MHz}$ ultrasound burst has a magnitude of $1 / 3$ of its fundamental frequency contribution. This implies that in the ultrasound-modulated light, both of the $5 \mathrm{MHz}$ and the $10 \mathrm{MHz}$ sidebands exist due to the two frequencies' contribution. When the two AOM's were tuned to burn two spectral holes at the $5 \mathrm{MHz}$ and 10 $\mathrm{MHz}$ sidebands, both components were filtered through the SHB crystal while the un-modulated light was strongly suppressed. The resulted amplitude of second harmonic UOT signal is $1 / 2$ of the fundamental UOT signal, indicting better filtering of the spectral hole for the higher harmonic.

A harmonic imaging result is shown in Figure 5. The sample was a $20-\mathrm{mm}$ thick phantom slab having the same composition as the 40-mm thick sample. Two absorbing objects were embedded in the middle plane of the phantom, with dimensions of $5 \times 5 \times 5 \mathrm{~mm}^{3}$ and $8 \times 8 \times 8 \mathrm{~mm}^{3}$, respectively. Their optical absorption coefficient is $\mu_{a}=10 \mathrm{~mm}^{-1}$ because of the addition of black India ink. Both of the fundamental image and the second harmonic image has adequate resolution and contrast to resolve the two absorbing inclusions. 

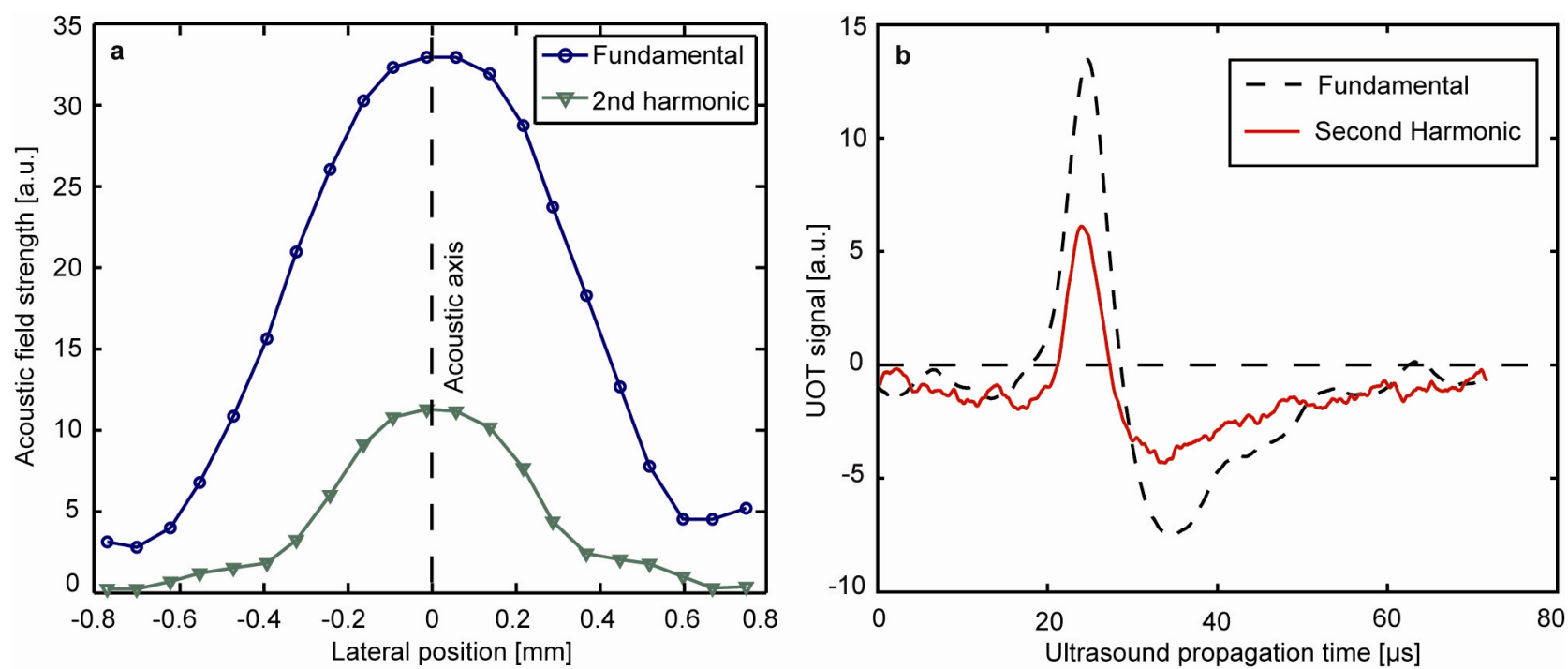

Figure 4a. Acoustic pressure contributed by the fundamental and second harmonic frequencies of the $5 \mathrm{MHz}$ focused transducer at its focus perpendicular to the ultrasound propagation direction. $4 \mathrm{~b}$. the detected UOT signals when a spectral-hole was burned at the fundamental or second harmonic frequency. The ultrasound burst consists of 5-cycle of $5 \mathrm{MHz}$ sinusoidal wave emitted by the ultrasound transducer whose focal pressure is $2 \mathrm{MPa}$.
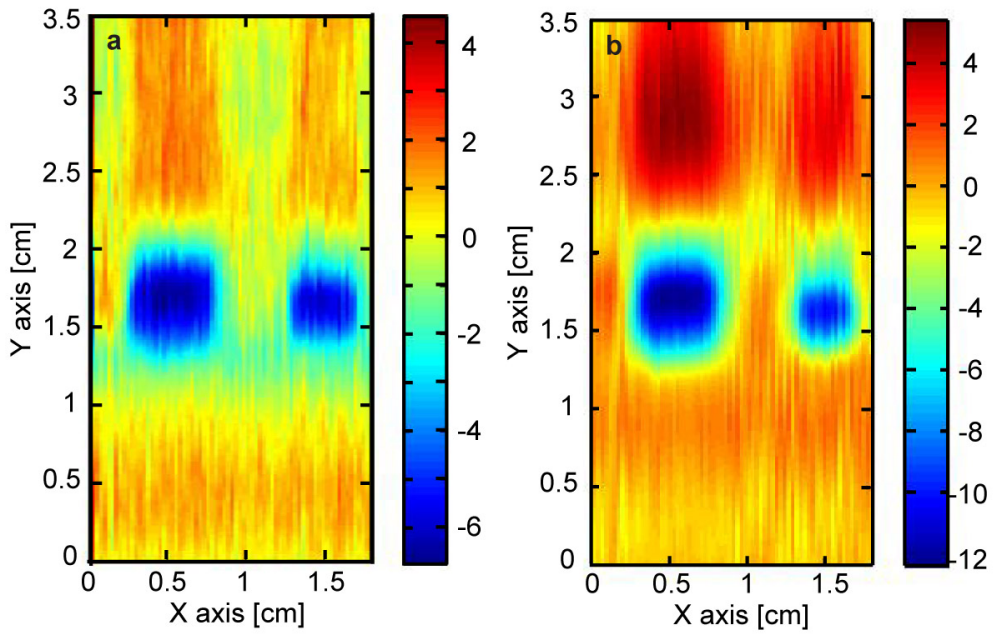

Figure 5. Harmonic image of two absorbing objects embedded in a 20-mm thick phantom sample. a. acquired when spectral holes were burned at the fundamental frequency; b. acquired when spectral holes were burned at the second harmonic frequency.

\section{FURTHER IMPROVEMENT OF SHB FOR UOT}

The signal detection enhancement by SHB for UOT as shown in the experimental results is very encouraging. The most recent SHB-UOT experiment result reported by other groups ${ }^{27}$ demonstrated an unprecedented imaging depth of $9 \mathrm{~cm}$ in tissue-mimicking phantom samples, which is close to practical applications, such as early breast cancer detection. However, there is still a lot of room to improve for this emerging technology. A lot of the improvement has to come from the development of a better SHB material that meets the following requirements: it needs to have a very high suppression to the un-modulated component, it needs to operate at a wavelength within the optical imaging window, it needs to have a longer life time and preferably works at higher temperatures. With the boost from the SHB detection, UOT will pave its way from research laboratories to everyday life. 


\section{ACKNOWLEDGEMENT}

This research has been funded in part by the National Institutes of Health grants R01 EB000712 and U54 CA136398.

\section{REFERENCES}

[1] Alfano, R. R. and Fujimoto, J. G., [Advances in Optical Imaging and Photon Migration (Vol. 2)], Optical Society of America, Washington, D. C., (1996).

[2] Cheong, W. F., Prahl, S. A. and Welch, A. J. "A Review of the Optical-Properties of Biological Tissues," IEEE J. Quant. Electron. 26, 2166-2185 (1990).

[3] Peters, V. G., Wyman, D. R., Patterson, M. S. and Frank, G. L., "Optical-Properties of Normal and Diseased Human Breast Tissues in the Visible and near-Infrared, " Phys Med. Biol. 35, 1317-1334 (1990).

[4] Troy, T. L., Page, D. L. and Sevick-Muraca, E. M., "Optical properties of normal and diseased breast tissues: prognosis for optical mammography, " J. Biomed. Opt. 1, 342-355 (1996).

[5] Mourant, J. R., Hielscher, A. H., Eick, A. A., Johnson, T. M. and Freyer, J. P., "Evidence of intrinsic differences in the light scattering properties of tumorigenic and nontumorigenic cells," Cancer Cytopathol. 84, 366-374 (1998).

[6] Santinelli, A., Baccarini, M., Colanzi, P. and Fabris, G., "Microvessel quantitation in intraductal and early invasive breast carcinomas," Anal. Quant. Cytol. Histol. 22, 277-284 (2000).

[7] Villringer, A. and Chance, B., "Non-invasive optical spectroscopy and imaging of human brain function," Trends Neurosciences 20, 435-442 (1997).

[8] Hebden, J. C. and Delpy, D. T., "Enhanced Time-Resolved Imaging with a Diffusion-Model of Photon Transport," Opt. Lett. 19, 311-313 (1994).

[9] Das, B. B., Yoo, K. M. and Alfano, R. R., "Ultrafast Time-Gated Imaging in Thick Tissues - a Step toward Optical Mammography," Opt. Lett. 18, 1092-1094 (1993).

[10] Marengo, S., Pepin, C., Goulet, T. and Houde, D., "Time-gated transillumination of objects in highly scattering media using a subpicosecond optical amplifier," IEEE J. Sel. Top. Quantum Electron. 5, 895-901 (1999).

[11] Fercher, A. F., Drexler, W., Hitzenberger, C. K. and Lasser, T., "Optical coherence tomography - principles and applications," Rep. Prog. Phys. 66, 239-303 (2003).

[12] Huang, D., et al. "Optical Coherence Tomography," Science 254, 1178-1181 (1991).

[13] Oraevsky, A. A., Jacques, S. L. and Tittel, F. K., "Measurement of tissue optical properties by time-resolved detection of laser-induced transient stress," Appl. Opt. 36, 402-415 (1997).

[14] Wang, X. D., et al. "Noninvasive laser-induced photoacoustic tomography for structural and functional in vivo imaging of the brain," Nature Biotech. 21, 803-806 (2003).

[15]Zhang, H. F., Maslov, K., Stoica, G. and Wang, L. H. V., "Functional photoacoustic microscopy for high-resolution and noninvasive in vivo imaging," Nature Biotech. 24, 848-851 (2006).

[16] Wang, L. H. and Zhao, X. M., "Ultrasound-modulated optical tomography of absorbing objects buried in dense tissue-simulating turbid media," Appl. Opt. 36, 7277-7282 (1997).

[17] Marks, F. A., Tomlinson, H. W. and Brooksby, G. W., "A comprehensive approach to breast cancer detection using light: Photon localization by ultrasound modulation and tissue characterization by spectral discrimination," Proc. SPIE 1888, 500-510 (1993)

[18] Leveque, S., Boccara, A. C., Lebec, M. and Saint-Jalmes, H., "Ultrasonic tagging of photon paths in scattering media: parallel speckle modulation processing," Opt. Lett. 24, 181-183 (1999).

[19] Sakadzic, S. and Wang, L. H. V., "High-resolution ultrasound-modulated optical tomography in biological tissues," Opt. Lett. 29, 2770-2772 (2004).

[20] Murray, T. W., et al. "Detection of ultrasound-modulated photons in diffuse media using the photorefractive effect," Opt. Lett. 29, 2509-2511 (2004).

[21] Leutz, W., and Maret, G., "Ultrasonic modulation of multiply scattered light," Physica B 204, 14-19 (1995).

[22] Kempe, M., Larionov, M., Zaslavsky, D. and Genack, A. Z., "Acousto-optic tomography with multiply scattered light," J. Opt. Soc. Am. A. 14, 1151-1158 (1997). 
[23] Li, Y. Z., Hemmer, P., Kim, C. H., Zhang, H. L. and Wang, L. H. V., "Detection of ultrasound-modulated diffuse photons using spectral-hole burning," Opt. Exp. 16, 14862-14874 (2008).

[24] Wang, L. H. V., "Mechanisms of ultrasonic modulation of multiply scattered coherent light: An analytic model," Phys. Rev. Lett. 87 043903(1-4) (2001).

[25] Sakadzic, S. \& Wang, L. H. V., "Ultrasonic modulation of multiply scattered coherent light: An analytical model for anisotropically scattering media," Phys. Rev. E 66 (2002).

[26] Gorju, G., Crozatier, V., Lorgere, I., Le Gouet, J. L. and Bretenaker, F. "10-GHz bandwidth RF spectral analyzer with $\mathrm{MHz}$ resolution based on spectral hole burning in Tm3+ : YAG," IEEE Photon. Tech. Lett. 17, 2385-2387 (2005).

[27]Zhang, H. L., et al., "Deep tissue (phantom) imaging with spectral hole burning in Pr : YSO," to be published. 